\title{
PSICOLOGÍA
}

\section{Nivel de relación y estimación de la eficacia predictiva de la personalidad, la inteligencia fluida y la situación laboral con respecto al rendimiento académico en estudiantes de una universidad privada de Asunción}

\author{
Mery Duarte, Jonathan Ayala
}

\begin{abstract}
Resumen
Introducción: La presente investigación consistió en identificar el nivel de predicción y relación de la personalidad, la inteligencia fluida y la situación laboral con respecto al rendimiento académico en estudiantes de una universidad privada de Asunción. El trabajo es un estudio de tipo descriptivo correlacional, de diseño no experimental el cual contó con una muestra de 155 personas seleccionadas por medio de muestreo no probabilístico por conveniencia de corte transversal, los instrumentos utilizados para la investigación se citan a continuación ;el Inventario de Personalidad NEO Revisado NEO PI-R de Costa y McCrae (1985), Test de Matrices Progresivas de Raven Avanzado creado por Raven (1938) y la Escala de situación laboral cuestionario auto aplicable creado, que presenta nueve ítems y evalúa la situación laboral usando los criterios planteados por Ibrahim, Freeman y Shelle (2011). Los resultados indican asociación entre los constructos: sentido del deber, responsabilidad, reflexión, impulsividad, modestia e inteligencia fluida en relación a rendimiento académico. Estos valores demuestran la existencia de asociación con efecto de tamaño mínimo entre los constructos mencionados. Finalmente, se elaboró un modelo formado por la faceta sentido del deber e inteligencia fluida, el cual explica el $15 \%$ de la varianza total del rendimiento académico.
\end{abstract}

Objetivo: Identificar el nivel de relación y estimar la eficacia predictiva de la personalidad, la inteligencia fluida y la situación laboral con respecto al rendimiento académico en estudiantes de una universidad privada de Asunción, identificar el nivel de relación entre facetas y factores de personalidad con respecto al rendimiento académico en estudiantes de una universidad privada de Asunción, determinar el grado de asociación entre inteligencia fluida y rendimiento académico en estudiantes de una universidad privada de Asunción,

1. Universidad del Cono Sur de las Américas, Paraguay.

E-mail: meryduarte17@hotmail.com

DOI: $10.26885 /$ rcei.foro.2019.239

Trabajo publicado en acceso abierto bajo Licencia Creative Commons. 
distinguir el nivel de relación entre situación laboral y rendimiento académico en estudiantes de una universidad privada de Asunción, identificar el nivel predictivo y combinado de los factores y las facetas de la personalidad, la inteligencia fluida y la situación laboral con respecto al rendimiento académico mediante regresión lineal múltiple en estudiantes de una universidad privada de Asunción.

Material y Método: Durante la recolección de datos se utilizó el inventario de Personalidad NEO Revisado NEO PI-R, test de Matrices Progresivas de Raven Avanzado, escala de situación laboral, calificaciones del rendimiento académico. El alcance del estudio fue descriptivo, correlacional y explicativo, de diseño no experimental el cual contó con una muestra total de 170 personas, que fueron seleccionadas mediante el muestreo no probabilístico por conveniencia.

Resultados: Los resultados indican correlación entre los constructos; sentido del deber $(r s=0,28 ; p=0,00)$, responsabilidad ( $r s=0,21 ; p=008)$, reflexión ( $r s=$ $0,21 ; p=0.08)$ impulsividad ( $r s=-0,19 ; p<0.15)$ modestia $(r s=0,18 ; p=0.22)$ e inteligencia fluida $(r s=0,18 ; p=0.22)$ en relación al rendimiento académico.

Conclusión: Primeramente, se concluye que existe asociación entre el factor responsabilidad de la personalidad con respecto al rendimiento académico; estos resultados confirman y refuerzan los hallazgos encontrados por Poropat (2009); Kappe y van der Flier (2010) y Peralta, Besio, Guzmán, y Henríquez (2015), por otra parte también se halló asociación con las facetas sentido del deber y reflexión que son facetas correspondientes al factor responsabilidad por lo que nuevamente se refuerzan las evidencias halladas por los autores mencionados.

Palabras clave: rendimiento académico, factores y facetas de la personalidad, inteligencia fluida, situación laboral.

\section{Referencias}

Academic Success for Part-Time Students at Polytechnic Institutes in Malaysia. International Journal of Adult Vocational Education and Technology, 2(4), 1.

Aguado, D. (2008). Analisis Inicial de las Propiedades Psicométricas del Cuestionario BFCP.

Aiken, L. (2003). Tests psicológicos y evaluación. México: Pearson Educación.

Allport, G. W. (1937). Personality: a psychological interpretation. New York: Holt, Rinehart, \& Winston.

Amador, J. A., Forns, M., \& Kirchner, T. (2005). Test de factor $g$ y factoriales: documentos de trabajo. Barcelona: Publicaciones Ediciones de la Universidad de Barcelona. 